\title{
APPLICATION OF GENETIC ALGORITHM FOR LIVER CANCER DETECTION
}

\author{
Yamini Upadhyay $^{1}$; Vikas Wasson ${ }^{2}$
}

\begin{abstract}
Human life is the most precious and valuable gift of God. The internal structure of human body is complex and weird. When there is any medical complication or problem it gets very important for doctor to analyze it correctly. X ray, CT and MRI images help doctor in this analysis. Cancer is a prominent reason for deaths in India. The cancer can be detected in human body through MRI images but it gets difficult to correctly analyze it due to the reason of having almost same intensity of the images of other body parts. A better system has to be find for the segmentation of liver from MRI images so that it can be identified clearly from the adjacent organs which have similar intensity. Also livers geometry is very complex due to its high anatomical variability both in health and disease. In this paper we will present different techniques for segmentation and will design a method for liver segmentation and cancer detection.
\end{abstract}

Keyword: Liver Segmentation, Genetic algorithm, MRI, Threshold based approaches. CBIR, Contour, Histogram

\section{INTRODUCTION}

There is a dire need to design and implement a quick responsive and exact calculative liver segmentation method for medical image analysis. For the need of computer aid in diagnoses, Liver segmentation is an important tool, it helps to evaluate the methodology and pros and cons of liver transplantation and the treatment method of liver tumors. Magnetic resonance imaging is comparatively a better method because it is free of ionizing radiation and also provides a good contrast visualization of soft tissue [2].There are a number of techniques for liver segmentation that include Region Growing, Threshold based, Level Set method, Statistical model, Active Contour, Clustering algorithm, Histogram based approach and Gray level methods, Genetic algorithm.

\section{LITERATURE SURVEY}

Due to the similar intensity of the organs and the complex geometry of the liver, it gets difficult to detect the cancer in the MRI of liver. There are also some artifacts of pulsation and motion, and partial volume effects due to different factors that make automatic liver segmentation difficult. The CT images show gray values in an abdominal image, which range between 90-92 out of 0-255 for a normal tumor free liver, whereas if there is tumor the range is not clear and the images show darker grey values. The pre existing methods which use the basic location and distribution location information of the general gray scale values of the image. There is a dire need to design and implement a quick responsive and exact calculative liver segmentation method for medical image analysis. For the need of computer aid in diagnoses, Liver segmentation is an important tool, it helps to evaluate the methodology and pros and cons of liver transplantation and the treatment method of liver tumors. Magnetic resonance imaging is comparatively a better method because it is free of ionizing radiation and also provides a good contrast visualization of soft tissue [2]

\subsection{Region based Approaches}

This method provides better results on contrast enhanced images. It uses a small region as seed point and progresses with the addition to the surrounding pixels, which will match the developed region in terms of the intensity. This process will end with the reception of the segmented area [3].

\subsection{Threshold based Approaches}

Due to the variation of liver intensity in CT images, it becomes difficult to use fixed threshold method. The liver segmentation using adaptive threshold technique should be used. The technique helps to use different threshold for different regions in the image [3].

\subsection{Level Set Approach}

This method can handle topological changes and define the problem in higher dimension, but this method is time consuming and results in over segmentation. The Segmentation using level set method that evolves according to a speed image that is the result of a scanning technique based dynamic programming. The main limitations is level set method adjusts this first segmentation using a speed function obtained from a pixel classification algorithm. The accuracy is only sufficient in a small number of cases [3].

\subsection{Model based Approach}

The statistical shape model based method has the best performance among all the approaches in the grand challenge 
workshop. This type of method uses Statistical Shape Model (SSM) that includes shape correspondence, shape representation, and search algorithms [3]. The drawback of the SSM method is that, it do not ensure better output until the dataset is large. Just in the same way, though most of the applications use contour based approach, but segmentation is for preprocessing, but when the content based image retrieval (CBIR) method is used, the time taken is more.

\subsection{Active Contour based Approach}

This image is based on the enhancing and denoising on the basis of filter. The filters can be based on chevshev or histogram equalization or anisotropic diffusion. It is based on the semi automatic segmentation of the liver for which different techniques can be used, GVF snake method is one of those techniques. Hermite-spline curve method is then implied to connect the manually selected points and thus the edges of the segmented area are designed using the edge detector [3].

\subsection{Gray Level based Approach}

An adaptive hybrid segmentation algorithm using Bayesian classification on volume intensities, the process is initiated by selecting any random pixel in the liver image. Of the adjoining rectangular area around this pixel the values of variance \& mean are determined. Then, a voxel classification with a smoothed MAP rule is applied to produce a segmentation label map [3].

\subsection{Generic Algorithm}

Genetic algorithms are optimization techniques inspired by natural evolution. In GA, solutions of an optimization problem are encoded as binary strings. After Generating a first set of random solutions, these can be improved by iteratively applying operators, termed selection, crossover and mutation, that mimic the corresponding processes of natural evolution. In fact, selection lets only the fittest individuals (best solutions, according to some goodness criteria or 'fitness function') be present in the next generation (iteration of the algorithm); crossover lets them exchange tracts of their DNA (corresponding substrings) to generate offspring (new solutions), while mutation randomly introduces new genes (by flipping one or more bits of a solution). Genetic algorithm (GA) is a computing model which mainly simulates biological heredity, mutation of evolutionary process in the nature and manifests thoughts through selection, crossover and mutation operators. Its main characteristics are the searching strategy, exchanging of information between individuals in a group. It is particularly appropriate for complex and nonlinear problems which were difficult to be resolve dealing with traditional methods, demonstrates its unique charm in combinatorial optimization, adaptive control, artificial life and other application areas. It is one of the intelligent computing technologies.

\section{PROBLEM DEFINITION AND OBJECTIVES}

It is important to detect and then analyse the root cell in the MRI images, so that the correct diagnosis and treatment can be decided. To implement this problem it is important to use segmentation of the liver images[7].This is the objective of the dissertation. Development of medical imaging technologies has made it a necessity to analyze patient datasets before taking any decision on treatment planning.

- The manual segmentation of the liver parenchyma is extremely laborious [10].

- Existing methods are cost intensive [5].

- Most of existing methods are time consuming [3].

\subsection{Objectives}

Development of medical imaging technologies has made it a necessity to analyze patient datasets before taking any decision on treatment planning. In case of liver, its size, volume, and shape; structure of its vessels; and tumors sizes and locations are important. The main objective includes:

- Acquire an image and perform pre-processing.

- Find Region of Interest (ROI) and perform segmentation with algorithm to be proposed.

- Compare performance of proposed algorithm with existing technique.

\section{METHODOLOGY}

In this review paper a technique for the segmentation of liver is to be opted. The methodology opted is that, a liver image will be selected, after that noise will be added to the image and then the image will be denoised. The region of interest(ROI) will be selected out of the denoised by making the fuzzy coded binary map. The binary map values are selected and their inverse is mixed with denoised values. These values are then given to an empty matrix, this results as segmented liver images. Out of the selected region of the segmented MRI image, using GA the cancer can be detected.

\subsection{Genetic Algorithm Based Clustering}

Genetic algorithm is based upon a simple principle of survival of the fittest. It uses clustering technique [11]. A cluster of pixels is taken. Genetic algorithm using a number of pixels is explained as:

1. First of all a chromosome is encoded using real, or binary numbers.

2. Then taking the values from the data set, clusters are defined and out of those the central cluster is initialized, which results as the population initialization.

3. Genetic algorithm represents the survival of the fittest. We define Davies Boulin index that is the ratio of sum of cluster separation within a cluster and between different clusters.

4. Then out of the existing population the fittest is selected to breed and generate the new generations. 
5. After this the crossover is done using stochastic method, from which the parents and child clusters are generated.

6. After which the valid and best fitted genes in chromosomes are mutated with a probability $\mu \mathrm{c}$

7. After a number of iterations, a suitable gene is preserved in a location outside the population with maximum fitness contains the centers of the final cluster.

\section{CONCLUSIONS}

The best opted method out of all the methods discussed in literature review is genetic algorithm, the choice may vary from user to user. The methodology explains that, a liver image will be selected, after that noise will be added to the image and then the image will be denoised. The region of interest(ROI) will be selected out of the denoised by making the fuzzy coded binary map. The binary map values are selected and their inverse is mixed with denoised values. These values are then given to an empty matrix, this results as segmented liver images. Out of the selected region of the segmented MRI image, using GA the cancer can be detected.

\section{REFERENCES}

[1] Pedro Rodrigues, Joao L. Vilaca and Jaime Fonseca, "An Image Processing Application for Liver Tumour Segmentation", IEEE, FEBRUARY 2011.

[2] Evgin Goceri, Mehmet Z. Unlu, Cuneyt Guzelis and Oguz Dicle, "An Automatic Level Set Based Liver Segmentation from MRI Data Sets", IEEE, 2012.

[3] S. Priyadarshni and Dr. D. Selvathi, "Survey on Segmentation of Liver from CT Images", IEEE, 2012.

[4] Angelina. S, L. Padma Suresh and S. H. Krishna Veni, "Image Segmentation Based On Genetic Algorithm for Region Growth and Region Merging”, IEEE, 2012.

[5] Ying Li, Yan-Ning Zhang, Ying-Lei Cheng, RongChun Zhao and Gui-Sheng Liao, "An Effective Method For Image Segmentation”, IEEE, 2005.

[6] S. Cagnoni, A. B. Dobrzeniecki, J. C. Yanch and R. Poli, "Interactive Segmentation of Multi-Dimensional Medical Data With Contour-Based Application of Genetic Algorithms", IEEE, 1994.

[7] Consuelo Cruz-Gomez, Petra Wiederhold and Marco Gudino-Zayas, "Automatic Liver Tissue Segmentation in Microscopic Images Using Fusion Color Space and Multiscale Morphological Reconstruction", IEEE, 2013.

[8] Jie Lu, Lin Shi, Min Deng, Simon C.H. YU and Pheng Ann Heng, "An Interactive Approoach To Liver Segmentation in CT Based on Deformable Model Integrated With Attractor Force", IEEE, 2011.

[9] Amir H. Foruzan, Yen-Wei Chen, Reza A. Zoroofi, Akira Furukawa, Yoshinobu Sato and Masatoshi Hori, "Multi-mode Narrow-band Thresholding with Application in Liver Segmentation from Low-contrast CT Images", IEEE, 2009.
[10] Jie Lu, Defeng Wang, Lin Shi and Pheng Ann Heng, "Automatic Liver Segmentation in CT Images based on Support Vector Machine", IEEE, 2012.

[11] Mridula J and Dipti Patra, "Genetic Algorithm based Segmentation of High Resolution Multispectral Images using GMRF Model", IEEE, 2010.

[12] Jiang Hua Wei and Yang Kai, "Research of Improved Genetic Algorithm for Thresholding Image Segmentation Based on Maximum Entropy", IEEE, 2010.

[13] S. S. Kumar and Dr. R. S. Moni, "Diagnosis of Liver Tumour from CT Images Using Fast Discrete Curvelet Transform", IJCA, 2010.

[14] N. Gopinath, " Extraction of Cancer Cells From MRI Prostate Image Using MATLAB", IJESIT, 2012.

[15] M. A. Ansari and R. S. Anand, "Region Based Segmenattion and Image Analysis with Application to Medical Imaging”, ICTES, 2007. 